\title{
Epidural Hematoma Related with Low-Dose Aspirin : Complete Recovery without Surgical Treatment
}

\author{
Kyoung-Tae Kim, M.D., ${ }^{1}$ Dae-Chul Cho, M.D., ${ }^{1}$ Suk-Won Ahn, M.D., ${ }^{2}$ Suk-Hyung Kang, M.D. ${ }^{3}$ \\ Department of Neurosurgery, ${ }^{\prime}$ Kyungpook National University Hospital, Daegu, Korea \\ Department of Neurology, ${ }^{2}$ College of Medicine, Chung-Ang University, Seoul, Korea \\ Department of Neurosurgery, ${ }^{3}$ Spine Center, Chuncheon Sacred Heart Hospital, Hallym University, Chuncheon, Korea
}

Hemorrhagic complications associated with aspirin use occur primarily at skin or gastrointestinal sites but can occasionally occur in the central nervous system. In particular, spontaneous spinal epidural hemorrhage (SSEH) associated with aspirin is very rare. We report a case of low-dose (100 mg daily) aspirin-related SSEH that was successfully treated with medical management. Our case indicates that low-dose aspirin could induce SSEH and that conservative treatment with close observation and repeated imaging studies should be considered in cases with neurological improvement or mild deficits.

Key Words : Aspirin · Spinal epidural hematoma · Medical management.

\section{INTRODUCTION}

Spontaneous spinal epidural hematoma (SSEH) is rare; however, it can present with neurological status change ranging from radiculopathy to complete quadriplegia depending on the severity of compression ${ }^{4}$. These hematomas have been reported in association with coagulopathies, anticoagulant therapy, tumor, infection, pregnancy and vascular malformations $s^{4,7-9)}$. However, no definite cause is found in the majority of cases ${ }^{4}$. Among these potential causative factors, anticoagulants may seem to be predictable ${ }^{16}$, but SSEH associated with anti-platelet agents is very rare. There are only six reported cases of aspirinrelated SSEH ${ }^{1,14,18,20,26,27)}$. In particular, the cases of SSEH related with low-dose aspirin are only two ${ }^{26,27}$. Here, we present a case of SSEH related with low-dose aspirin that was successfully treated without surgery.

\section{CASE REPORT}

A 50-year-old man visited the emergency room complaining of severe neck pain and quadriparesis [American Spinal Injury Association (ASIA) impairment scale : C]. He had no history of trauma or spinal pain. He had been taking aspirin (100 mg daily) without medical advice or prescription for four months. General laboratory findings were normal, but platelet aggregation time was slightly prolonged. Bleeding time (Collagen/Epinephrine clotting time) was $230 \mathrm{sec}$, which was slightly delayed (normal range : $81-192 \mathrm{sec}$ ). Initial spine computerized tomography (CT) showed a high-density mass in the dorsal portion of the epidural space from C3 to C5 (Fig. 1A). He was prescribed a neck brace and steroid therapy (dexamethasone $5 \mathrm{mg}$ IV) with a prophylactic antacid agent. One hour later, partial improvement was observed in his neurological status (ASIA impairment scale : $\mathrm{C} \rightarrow \mathrm{D}$ ), and we were able to safely obtain a spinal magnetic resonance image (MRI). MRI showed a large hematoma in the dorsal portion of the epidural space from $\mathrm{C} 3$ to $\mathrm{C} 5$, causing spinal cord compression (Fig. 1B). Three hours later, the patient demonstrated normal motor function with mild numbness in both arms. Therefore, we continued conservative treatment (brace and steroid) in the intensive care unit and performed a follow-

- Received : August 2, 2011 • Revised : February 6, 2012 • Accepted : May 15, 2012

- Address for reprints : Suk-Hyung Kang, M.D.

Department of Neurosurgery, Spine Center, Chuncheon Sacred Heart Hospital, Hallym University, 77 Sakju-ro, Chuncheon 200-704, Korea

Tel : +82-33-240-5173, Fax : +82-33-255-6244, E-mail : nscharisma@hanmail.net

- This is an Open Access article distributed under the terms of the Creative Commons Attribution Non-Commercial License (http://creativecommons.org/licenses/by-nc/3.0)

which permits unrestricted non-commercial use, distribution, and reproduction in any medium, provided the original work is properly cited. 
up CT. Follow-up CT one day later showed a partial decrease in the hematoma (Fig. 1C), and his neurological deficits had fully recovered. Spinal artery digital substraction angiography was performed to rule out vascular lesion, and there was no abnormality. Seven days later, a follow-up MRI showed complete resolution of the epidural hematoma (Fig. 1D) and enhanced MR images showed no abnormal enhancing lesion, such as tumor or vascular malformation. The patient was discharged without neurological deficit.

\section{DISCUSSION}

SSEH is a relatively rare disease : its incidence is 0.1 patients per 100000 , representing $<1 \%$ of spinal epidural space occupying lesions ${ }^{17)}$. The main causes include vascular malformation, anticoagulant therapy, and neoplasm ${ }^{7,8,13,20,26)}$. Of these potential causative factors, anticoagulants may be predictable : it has been reported that $25-70 \%$ of SSEH patients have a history of anticoagulant use ${ }^{16)}$. However, SSEH associated with anti-platelet agents is very rare.

Anti-platelet drug is frequently used in various diseases, and their hemorrhagic complications usually occur at skin or gastrointestinal sites ${ }^{15,19)}$. In particular, aspirin is one of the most widely used medications in the world, with approximately 40000 ton consumed each year ${ }^{27}$ ). Aspirin (also known as acetylsalicylic acid) is used for its analgesic, antipyretic, and anti-inflammatory properties. Aspirin also has antiplatelet effects due to inhibition of thromboxane production; thus, it is used long-term at low doses to help prevent heart attacks, ischemic strokes, and blood clot formation in patients at high risk for clot development ${ }^{15)}$. However, there are also well-established undesirable side effects of aspirin, including stomach bleeding, gastrointestinal ulcers, tinnitus, and hemorrhagic stroke especially at higher doses ${ }^{15,19)}$.

There have been six reported cases of aspirin-related SSEH ${ }^{1,14,18,20,26,28)}$ and only two cases of low-dose aspirin-related SSEH ${ }^{26,28)}$. The CURRENT-OASIS 7 study reported that occurrences of major bleeding, such as gastrointestinal bleeding and intracranial hemorrhage, did not differ between high-dose (300-325 mg daily) and low-dose aspirin (75-100 mg daily) ${ }^{19)}$. Wagner et al. ${ }^{26)}$ re- ported low-dose aspirin induced SSEH, which showed moderate coagulopathy including of $10-16 \%$ platelet aggregation with arachidonic acid and collagen (normal range : $>70 \%$ ) and normal bleeding time. In our case, platelet aggregation and bleeding time were slightly delayed, so we cannot completely rule out the possibility of a coincidence of the medical history of aspirin intake and SSEH. However, significant numbers of the patients with secondary intracranial hemorrhage related with low-dose aspirin showed normal or mild abnormal platelet function and bleeding time $\mathrm{e}^{20,21,25)}$. Although the SSEH is different from the intracranial hemorrhage, but the probability of aspirin-related SSEH would seem to be higher considering its action mechanism and patient's radiologic and lab findings.

Immediate surgical decompression of the neural structure is the treatment of choice in the majority of patients, but conservative management has occasionally been considered ${ }^{11,12)}$. Decision to treat conservatively is based on the neurological status and surgical condition of the patient. Neurological improvement and mild neurological deficit without progression are good indications for conservative treatment ${ }^{12,17)}$. Coexisting serious coagulopathy and/or anticipated risks of operative treatment may also be relative indications for conservative treatment ${ }^{10,23}$. However, there are some debates regarding the methods of conservative treatment.

Some authors suggested that immediate replacement therapy in patients with a coagulopathy prevents progression of the hematoma, allowing for the improvement of neurological signs and symptoms without operation ${ }^{10,24)}$. In contrast, Connolly et al. ${ }^{5)}$ stated that coagulopathy-induced spinal bleeds are amendable to conservative treatment because the hematoma remains liquid for a longer time compared to that in cases with normal clotting, therefore enabling the spread of the hematoma into the spinal epidural space. Crabbe suggested that immobilization of the neck and administration of steroids may result in rapid improvement of neurological deficits in cervical $\mathrm{SSEH}^{6}$, and $\mathrm{Fu}$ kui et al..$^{9)}$ reported that cervical/cervicothoracic localization favors spontaneous recovery after SSEH.

However, it is clear that intensive neurologic examination and follow-up imaging studies are necessary during conservative treatment. In our case, we chose neck immobilization and ste-
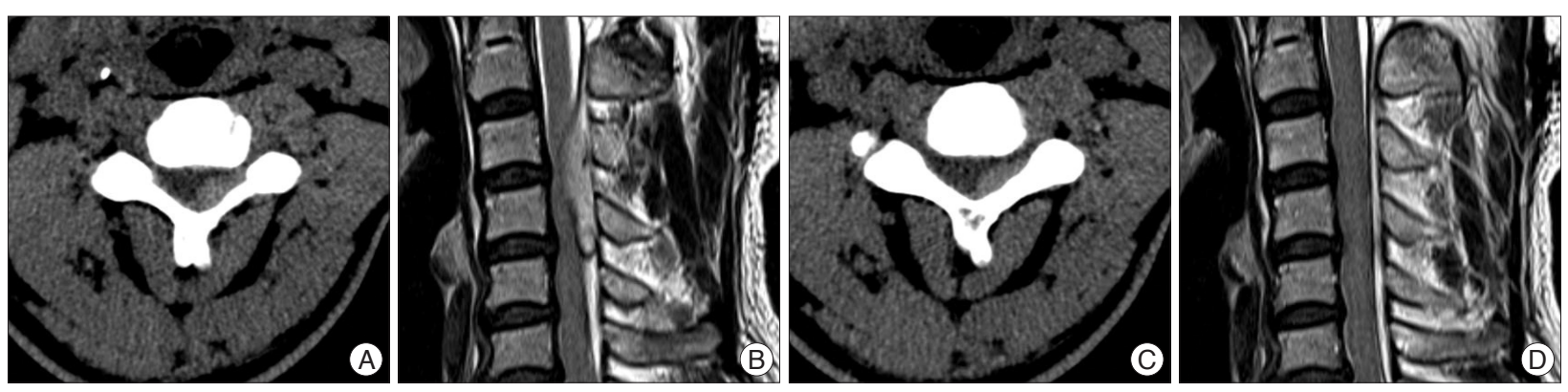

Fig. 1. Initial and follow-up imaging studies. A : Initial cervical CT shows a high density mass in the dorsal portion of the epidural space from C3 to C5. B : Initial T2 sagittal cervical MRI shows a huge mass in the dorsal portion of the epidural space from C3 to C5 with cord compression. C : Twenty-four hour follow-up CT shows a partial decrease of the hematoma. D : Seven day follow-up MRI (T2 sagittal image) shows complete resolution of the epidural hematoma. 
roid administration, which was shown to have a rapid response and excellent outcome. Recovery or dramatic improvement of clinical symptoms without surgery is not common, although there are some reports ${ }^{1,3,5,11,24,26)}$. We performed follow-up CT one day later and MRI seven days later. Although his neurological deficits had fully recovered after one day, we need a followup imaging study for precise care. MRI is the best diagnostic modality ${ }^{11)}$, but it requires a longer study time and has a high cost compared to CT. CT is also a good follow-up modality ${ }^{1)}$, so we performed a follow-up CT after one day.

There has been much speculation on the cause of spontaneous resolution of neurological signs and symptoms after SSEH. Among the various theories, the widely known "spreading theory" which suggests spread of the hematoma within the spinal canal along the spinal epidural space, is a plausible explanation for spontaneous recovery ${ }^{11,12)}$. Groen suggested that spread of a hematoma remains possible until blood clotting is complete and that delayed clot formation (due to anticoagulants or coagulopathies) may promote spreading of the hematoma ${ }^{2,11)}$.

In our case, the neurological deficits had fully recovered at 24 hours; by the seventh day, follow-up MRI showed complete resolution of the epidural hematoma. Wagner et al. reported a case of aspirin-related SSEH that had completely resolved on day 3 as seen on MRI. These rapid recoveries are meaningful considering that other reports have noted recovery times of six days to two months ${ }^{2,11}$. Although there have been only two cases of rapid resolutions, they may be explained by Groen's description ${ }^{2,11}$.

\section{CONCLUSION}

The use of aspirin in Korea continues to become more widespread with the increase in the elderly population ${ }^{22}$. Although we could not find clinical data regarding the use of low-dose aspirin in Korea, it is believed to be widely used. Our case indicates that low-dose aspirin can induce SSEH and physicians should be aware of this rare but serious complication. Also, conservative treatment involving close observation and repeated imaging studies may be considered in those with neurological improvements or mild deficits.

To our knowledge, this is the first Korean case of SSEH related with low-dose aspirin that was successfully treated without surgical treatment.

\section{References}

1. Anderson TJ, Donaldson IM : Spontaneous resolution of cervical spinal epidural haematoma. Postgrad Med J 65 : 488-490, 1989

2. Boukobza M, Guichard JP, Boissonet M, George B, Reizine D, Gelbert F, et al. : Spinal epidural haematoma : report of 11 cases and review of the literature. Neuroradiology 36 : 456-459, 1994

3. Brawn LA, Bergval UE, Davies-Jones GA : Spontaneous spinal epidural haematoma with spontaneous resolution. Postgrad Med J 62 : 885-887, 1986

4. Bruyn GW, Bosma NJ : Spinal extradural haematoma in Vin-ken PJ, Bruyn GW (eds) : Handbook of Clinical Neurology. Amsterdam :
North-Holland, 1976, Vol 26, pp1-30

5. Connolly ES Jr, Winfree CJ, McCormick PC : Management of spinal epidural hematoma after tissue plasminogen activator. A case report. Spine (Phila Pa 1976) 21 : 1694-1698, 1996

6. Crabbe DC, Mendelow AD, Pharoh P, Large DM, Ions GK : Cervical spinal extradural haematoma causing a transient Brown-Sequard syndrome. J Neurol Neurosurg Psychiatry 55 : 239, 1992

7. Foo D : Spinal epidural hematoma. J Neurosurg $84: 308,1996$

8. Foo D, Chang YC, Rossier AB : Spontaneous cervical epidural hemorrhage, anterior cord syndrome, and familial vascular malformation. Neurology 30 : 1253-1254, 1980

9. Fukui MB, Swarnkar AS, Williams RL : Acute spontaneous spinal epidural hematomas. AJNR Am J Neuroradiol 20 : 1365-1372, 1999

10. García López A, Pérez Lara JM, Herrainz Hidalgo R, Puente Gonzalo E : Spinal epidural hematoma following thrombolytic therapy for acute myocardial infarction. Orthopedics 22 : 987-988, 1999

11. Groen RJ : Non-operative treatment of spontaneous spinal epidural hematomas : a review of the literature and a comparison with operative cases. Acta Neurochir (Wien) $146: 103-110,2004$

12. Halim TA, Nigam V, Tandon V, Chhabra HS : Spontaneous cervical epidural hematoma : report of a case managed conservatively. Indian J Orthop $42: 357-359,2008$

13. Harris DJ, Fornasier VL, Livingston KE : Hemangiopericytoma of the spinal canal. Report of three cases. J Neurosurg 49 : 914-920, 1978

14. Heye $\mathrm{N}$ : Is there a link between acute spinal epidural hematoma and aspirin? Spine (Phila Pa 1976) 20 : 1931-1932, 1995

15. Lewis HD Jr, Davis JW, Archibald DG, Steinke WE, Smitherman TC, Doherty JE 3rd, et al. : Protective effects of aspirin against acute myocardial infarction and death in men with unstable angina. Results of a Veterans Administration Cooperative Study. N Engl J Med 309 : 396-403, 1983

16. Liao CC, Lee ST, Hsu WC, Chen LR, Lui TN, Lee SC : Experience in the surgical management of spontaneous spinal epidural hematoma. J Neurosurg $100: 38-45,2004$

17. Liu Z, Jiao Q, Xu J, Wang X, Li S, You C : Spontaneous spinal epidural hematoma : analysis of 23 cases. Surg Neurol 69 : 253-260; discussion 260, 2008

18. Locke GE, Giorgio AJ, Biggers SL Jr, Johnson AP, Salem F : Acute spinal epidural hematoma secondary to aspirin-induced prolonged bleeding. Surg Neurol $5: 293-296,1976$

19. Mehta SR, Tanguay JF, Eikelboom JW, Jolly SS, Joyner CD, Granger CB, et al. : Double-dose versus standard-dose clopidogrel and high-dose versus low-dose aspirin in individuals undergoing percutaneous coronary intervention for acute coronary syndromes (CURRENT-OASIS 7) : a randomised factorial trial. Lancet $376: 1233-1243,2010$

20. Mishima K, Aritake K, Morita A, Miyagawa N, Segawa H, Sano K : [A case of acute spinal epidural hematoma in a patient with antiplatelet therapy]. No Shinkei Geka 17 : 849-853, 1989

21. Ohm C, Mina A, Howells G, Bair H, Bendick P : Effects of antiplatelet agents on outcomes for elderly patients with traumatic intracranial hemorrhage. J Trauma 58 : 518-522, 2005

22. Park IB, Kim DJ, Kim J, Kim H, Kim H, Min KW, et al. : Current status of aspirin user in Korean diabetic patients using Korean health insurance database. J Korean Diabetes Assoc 30 : 363-371, 2006

23. Rois PV, López MR, de Vergara BC, de la Lama Zaragoza A, García JG, Uxo JM : Spinal epidural hematoma in hemophilic children : controversies in management. Childs Nerv Syst 25 : 987-991; discussion 993, 995, 2009

24. Schmitz A, Wallny T, Sommer T, Brackmann H, Schulze-Bertelsbeck D, Effenberger W, et al. : Spinal epidural haematoma in haemophilia A. Haemophilia 4 : 51-55, 1998

25. Tauber M, Koller H, Moroder P, Hitzl W, Resch H : Secondary intracra- 
nial hemorrhage after mild head injury in patients with low-dose acetylsalicylate acid prophylaxis. J Trauma 67 : 521-525; discussion 525, 2009

26. Wagner S, Forsting M, Hacke W : Spontaneous resolution of a large spinal epidural hematoma : case report. Neurosurgery 38 : 816-818, 1996

27. Warner TD, Mitchell JA : Cyclooxygenase-3 (COX-3) : filling in the gaps toward a COX continuum? Proc Natl Acad Sci USA 99 : 1337113373,2002

28. Weber J, Hoch A, Kilisek L, Spring A : [Spontaneous intraspinal epidural hematoma secondary to use of platelet aggregation inhibitors]. Dtsch Med Wochenschr 126 : 876-878, 2001 